\title{
A Modified Lax-Friedrichs Method for the Shallow Water Equations
}

\author{
Kartika Yulianti ${ }^{1}$, Rini Marwati ${ }^{2}$, Suci Permatahati ${ }^{3}$ \\ $\left\{\right.$ kartika.yulianti@upi.edu ${ }^{1}$,rinimarwati@upi.edu² $\}$ \\ Department of Mathematics Education, Universitas Pendidikan Indonesia (UPI), \\ Jl. Dr. Setiabudi no 229 Bandung 40154, Indonesia ${ }^{1,2,3}$
}

\begin{abstract}
In this paper, we present a simple modified Lax-Friedrichs method for the numerical solution of shallow water equations. In the proposed method, we approximate the next-step value by applying the Lax-Friedrichs method at the half grid of space. The method was applied to simulate the wave propagation at a resting pool for non-flat topography. Based on the simulation, the proposed method is more accurate in terms of fulfilling the conservation law than the Lax-Friedrichs method and it shows good agreement with the steady analytical solution.
\end{abstract}

Keywords: Shallow water equations, Lax-Friedrichs method, Modification

\section{Introduction}

The shallow water equations (SWE) are nonlinear hyperbolic systems of partial differential equations that consist of simplifying the conservative equation and the momentum equation. The simplification came as a consequence from the assumption that on the SWE the horizontal length scale is much greater than the depth scale. Thus the considered velocity component is on horizontal direction only. This equation has been widely used to model phenomena that involve wave motion, such as water flows, flood waves, tsunami waves, and dam-break problems.

Many numerical methods are available in the literature to solve the SWE, such as the finite difference method, the Lax-Friedrichs method, the Lax-Wendroff Method, the Upwind Methods, and the Godunov's Method. On its extension, the researcher created and improved the methods to produce a more accurate and more efficient solution. Saiduzzaman and Ray compared the solution of the shallow water equation by different schemes, i.e Lax-Friedrichs scheme, LaxWendroff scheme, and Leap-Frog scheme [1]. They stated that the error term in the Lax-Wendroff scheme is less than the Lax-Friedrichs scheme and the Leap-Frog scheme. Benkhaldoun and Seaïd proposed a method for the numerical solution of SWE which is consists of a predictor stage and a corrector stage [2]. Doyen and Gunawan developed an explicit finite volume scheme for the SWE, where the upwind and the centered discretizations are used to compute numerical fluxes [3]. August et. al. derived a well-balanced scheme to overcome inaccurate numerical solution problem for near steady states that caused by numerical truncation errors [4].

The Lax-Friedrichs method is a classical explicit three-point scheme to solve the SWE. The method is simple to implement. However, this method introduces much more diffusion than is required and gives numerical results that are typically badly smeared unless the grid is very fine [5]. Boukadida and Leroux developed a two-dimensional version of the Lax-Friedrichs 
Scheme by using corrected fluxes to reduce diffusion in the scheme [6]. Press studied the structure of the operator corresponding to the Lax-Friedrichs method and gave a recipe for better solutions [7]. Lu et. al. proposed two time-step-convergence improved schemes to resolve the excessive numerical diffusion [8].

In this paper, we propose a simple modification of the Lax-Friedrichs scheme to reduce the diffusion in the scheme by defining the average term at the half grid of space. To test the accuracy, the results of the proposed scheme are then verified to the analytical steady-state solution of SWE at a resting pool.

\section{The Modified Lax-Friedrichs Method}

We consider a homogeneous hyperbolic equation which is called conservation law:

$$
q_{t}(x, t)+f(q(x, t))_{x}=0
$$

where $f(q)$ is the flux function. Let $Q_{i}^{n}$ is the approximation of the average solution $q$ over the $i$ th interval at the time $t_{n}$ :

$$
Q_{i}^{n} \approx \frac{1}{\Delta x} \int_{x_{i-\frac{1}{2}}}^{x} x_{i+\frac{1}{2}} q\left(x, t_{n}\right) d x
$$

with $\Delta x=x_{i+\frac{1}{2}}-x_{i-\frac{1}{2}}$. Based on Leveque [5], the Lax-Friedrichs scheme for the solution of equation (1) has the form:

$$
Q_{i}^{n+1}=\frac{1}{2}\left(Q_{i-1}^{n}+Q_{i+1}^{n}\right)-\frac{\Delta t}{2 \Delta x}\left[f\left(Q_{i+1}^{n}\right)-f\left(Q_{i-1}^{n}\right)\right]
$$

The Lax-Friedrichs method is stable for $v \leq 1$, where $v$ is the Courant number. According to Leveque [5], if the term average $\frac{1}{2}\left(Q_{i-1}^{n}+Q_{i+1}^{n}\right)$ of the equation (2) is replaced by $Q_{i}^{n}$ then the scheme is unstable. So, the average term acts as a damp for the instability. In this paper, we propose the modified Lax-Friedrichs by increase the order of instability damped as follow:

$$
Q_{i}^{n+1}=\frac{1}{4}\left(Q_{i-1}^{n}+2 Q_{i}^{n}+Q_{i+1}^{n}\right)-\frac{\Delta t}{2 \Delta x}\left[f\left(Q_{i+1}^{n}\right)-f\left(Q_{i-1}^{n}\right)\right]
$$

To apply to the shallow water equation, the SWE can be written in a conservative form as

$$
\partial_{t}\left(\begin{array}{c}
h \\
h u
\end{array}\right)+\partial_{x}\left(\begin{array}{c}
h u \\
h u^{2}+\frac{1}{2} g h^{2}
\end{array}\right)=\left(\begin{array}{c}
0 \\
-g h \partial_{x} Z
\end{array}\right) .
$$

where $h(x, t)$ is the height of the water, $u(x, t)$ is the flow velocity, $g$ is the gravity, and $Z(x)$ is the function for the bottom topography. Then the modified Lax-Friedrichs scheme for the shallow water equation is: 


$$
\begin{gathered}
h_{i}^{n+1}=\frac{1}{4}\left(h_{i-1}^{n}+2 h_{i}^{n}+h_{i+1}^{n}\right)-\frac{\Delta t}{2 \Delta x}\left[h_{i+1}^{n} u_{i+1}^{n}-h_{i-1}^{n} u_{i-1}^{n}\right] \\
u_{i}^{n+1} h_{i}^{n+1}=\frac{1}{4}\left(u_{i-1}^{n} h_{i-1}^{n}+2 u_{i}^{n} h_{i}^{n}+u_{i+1}^{n} h_{i+1}^{n}\right)-\frac{\Delta t}{2 \Delta x}\left[h_{i+1}^{n}\left(u_{i+1}^{n}\right)^{2}+\frac{1}{2} g\left(h_{i+1}^{n}\right)^{2}-\right. \\
\left.h_{i-1}^{n}\left(u_{i-1}^{n}\right)^{2}-\frac{1}{2} g\left(h_{i-1}^{n}\right)^{2}\right]-\frac{\Delta t}{2 \Delta x}\left[g h_{i}^{n}\left(Z_{i+1}^{n}-Z_{i-1}^{n}\right)\right] .
\end{gathered}
$$

\section{Simulation of SWE at a resting pool and its verification}

In subsection 3.1, we show the numerical results for wave propagation at a resting pool. In subsection 3.2, the numerical solutions are verified with the analytical solutions at steady-state conditions. The solutions resulted from the modified Lax-Friedrichs method also compared to the classical Lax-Friedrichs method.

\subsection{Numerical Results}

For simulation, we consider the computational domain $x \in[-2,2], t \in[0,22]$ and the mesh width $\Delta x=0.1, \Delta t=0.01$. To represent the condition at the walls of the pool, we set the boundary conditions:

$$
\begin{aligned}
& u_{x}( \pm 2,0)=0, \\
& h_{x}( \pm 2,0)=0 .
\end{aligned}
$$

For the function of topography and the initial conditions we defined:

$$
\begin{aligned}
& Z(x)=-0.02(x-2), \\
& u(x, 0)=2, \\
& h(x, 0)=1+0.1 \exp \left(1-x^{2}\right)-Z(x) .
\end{aligned}
$$

In Figures 1 and 2, we show numerical results of our method for the height of the water and flow velocity, respectively. Based on Figure 1, it can be shown that from the initial height, the water oscillates. The amplitude monotonically decreases. At $t=10$ and $t=22$, the oscillation is very small. In other words, they almost attain steady-state conditions, i.e. the constant height. From this simulation, the constant steady solution is $h(x, 22) \approx h(x) \approx$ 1.1159 


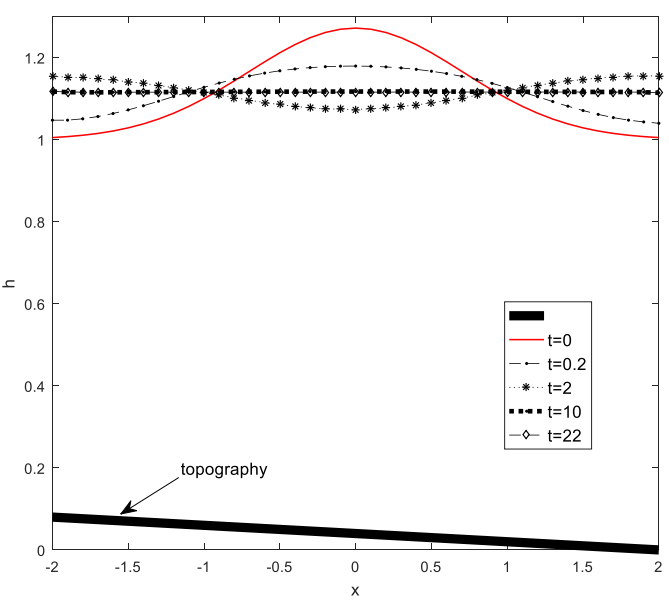

Fig. 1. Height of the water.

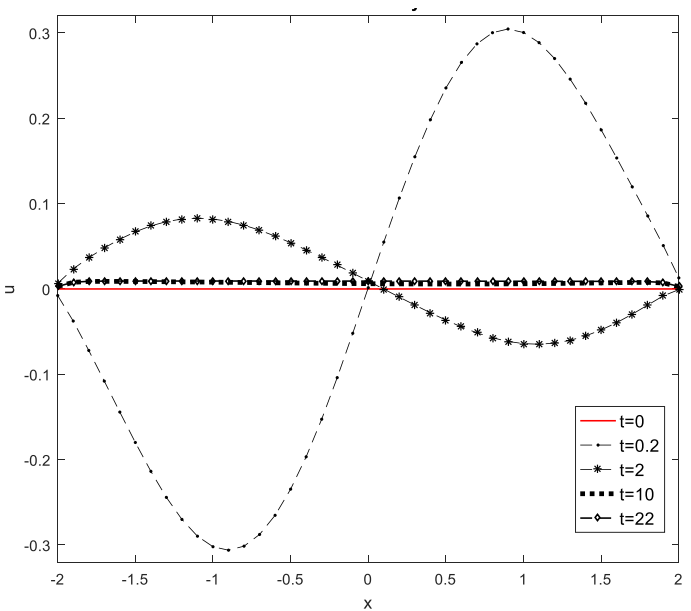

Fig. 2. Flow velocity.

The flow velocity for the wave several times is presented in Figure 2. In this simulation, initially, the velocity is zero. Then at $t=0.2$, for $x>0$ the velocity is positive, and vice versa for $x<0$. It means that for $x>0$ the wave moves in the right direction, and for $x<0$ it moves to the left direction. Hereafter, the values of the velocity decrease and the signs are reversed. This situation corresponds to the oscillating wave with decreased amplitude. The flow velocities at $t=10$ and $t=22$ are not significantly different. Both values tend to zero, heading to the steady-state condition. We remark here that the steady-state condition is achieved when $u(x, t)=0$.

\subsection{Accuracy Tests}


An important property reflecting the accuracy of our simulations is the conservation of mass. Since at the boundary conditions there is no either flux out of flux in, then the total mass along the simulations must be conserved. To compare the accuracy of our modified LaxFriedrichs scheme to the classical Lax-Fridrich scheme, we calculated the total area $(A)$ along with the simulations. Based on the initial height, the initial area $\left(A_{\text {ref }}\right)$ is 4.3195 . The area at $t$ $=22$ for the modified Lax-Fridrich is 4.3034, whereas for the classical Lax-Fridrich is 4.2923. Thus, the modified Lax-Fridrich method is more conserved than the classical Lax-Fridrich method.

To test the accuracy, we also verify the numerical solution to the analytical solution at a steady-state condition. The analytical steady-state solutions are obtained when $\partial_{t} h=0$ and $\partial_{t}(h u)=0$. It is expected that the steady-state solution for water velocity $\left(u_{r e f}\right)$ is zero. For the case function of topography is linear $\left(\partial_{x} Z\right.$ is a constant $\left.P\right)$, the steady-state solution for $h(x)$ can be obtained from:

$$
\partial_{x}\left(\frac{1}{2} g h^{2}\right)=-g h P
$$

So that, $h(x)=-P x+c$, where $c$ is a constant that associates with the area between the water free-surface and the topography. Since the initial area is 4.3195 and $P=-0.02$, then the value of $c$ is 1.08 . Thus the steady-state solution for water height is

$$
h(x)=0.02 x+1.08
$$

The water free-surface is represented by $h(x)+Z(x)$. As a result, we have a constant freesurface of water $\left(h_{r e f}\right)$ is 1.12 .

The comparisons errors for the two methods are presented in Table 1. From the table, it can be seen that the modified Lax-Friedrichs method is more accurate than the classical Lax-Fridrich method.

Table 1. The comparisons errors for the two methods.

\begin{tabular}{lcll}
\hline & & $\begin{array}{l}\text { The modified } \\
\text { Lax-Friedrichs method }\end{array}$ & $\begin{array}{l}\text { The classical } \\
\text { Lax-Friedrichs method }\end{array}$ \\
\hline \multirow{2}{*}{ Total mass } & $\left|A-A_{\text {ref }}\right|$ & 0.0161 & 0.0272 \\
& Error $(\%)$ & 0.3731 & 0.6308 \\
\hline \multirow{2}{*}{ Steady height } & $\left|h-h_{\text {ref }}\right|$ & 0.004 & 0.007 \\
& Error $(\%)$ & 0.357 & 0.625 \\
\hline Steady velocity & $\left|u-u_{\text {ref }}\right|$ & 0.0088 & 0.0143 \\
\hline
\end{tabular}

\section{Conclusion}

In this paper, we developed a method for the numerical solution of the Shallow Water Equation. The method is a modification of the Lax-Friedrichs method by replacing the average term which acts as a damped instability with the average term in higher order. The new method has been tested to simulate the wave propagation at a resting pool with non-flat topography. 
Based on the simulations, the new method is more accurate than the Lax-Friedrichs method in terms of the mass conservation principle and analytical steady-state solution.

Acknowledgments. The paper is funded by grant research from UPI.

\section{References}

[1] Saiduzzaman, M. \& Ray, S. K.: Comparison of Numerical Schemes for Shallow Water Equation. Global Journal of Science Frontier Research. Vol 13 (2013)

[2] Benkhaldoun, F., and Seaïd, M.: A simple finite volume method for the shallow water equations. Journal of Computational and Applied Mathematics. Vol. 234 pp. 58-72 (2010)

[3] Doyen and Gunawan, P.H.: An Explicit Staggered Finite Volume Scheme for the Shallow Water Equations. In: Finite Volumes for Complex Applications VII-Methods and Theoretical Aspects. Springer, pp. 227-235 (2014)

[4] Audusse, E, Bouchut, F., Bristeau, M., Klein, R., and Perthame, B.: A Fast and Stable WellBalanced Scheme with Hydrostatic Reconstruction for Shallow Water Flows. SIAM Journal on Scientific Computing. Vol. 25, No. 6, pp. 2050-2065 (2004).

[5] Leveque, R. J. : Finite Volume Methods for Hyperbolic Problems, Cambridge University Press (2004)

[6] Boukadida, T. and Leroux, A.Y.: A New Version of the Two-Dimensional Lax-Friedrichs Scheme. Mathematics of Computation. Vol 63, no. 208 pp. 541-553 (1994)

[7] Breuss, M.: The Correct Use of the Lax-Friedrichs Method. ESAIM: Mathematical Modelling and Numerical Analysis. Vol 38, No. 3, pp. 519-540 (2004)

[8] Lu, X., Dong, B., Mao, B., Zhang, X.: Convergence Improved Lax-Friedrichs Scheme Based Numerical Schemes and Their Applications in Solving the One-Layer and Two-Layer Shallow-Water Equations. Mathematical Problems in Engineering. Vol 2015. (2015) 